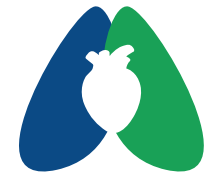

ASSOBRAFIR C I Ê N C I A

\title{
Barreiras à adesão ao programa de reabilitação pulmonar de pacientes com doença pulmonar obstrutiva crônica
}

\author{
Barriers to adherence to the pulmonary rehabilitation program of \\ patients with chronic obstructive pulmonary disease
}

Carolina Lima da Fonte ${ }^{*}$ (D), Beatriz Brito Gomes ${ }^{1}$ (D) , Soany de Jesus Valente Cruz ${ }^{2}$ (D), William Rafael Almeida Moraes ${ }^{2}$ (1), Laura Maria Tomazi Neves ${ }^{2,3}$ (D)

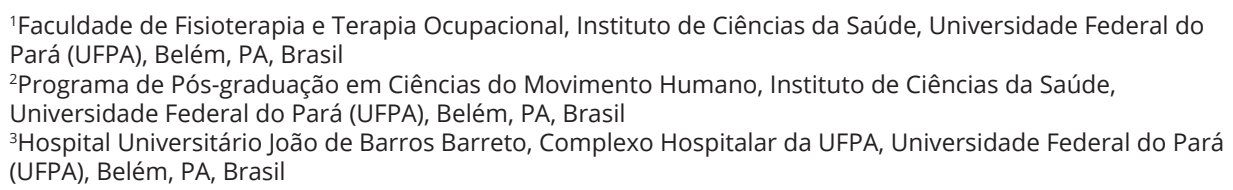

\section{Resumo}

Introdução: A reabilitação pulmonar é de suma importância para pacientes com DPOC. Entretanto, a adesão ao tratamento é baixa. Compreender os motivos da baixa adesão é imprescindível para o sucesso da terapia. Objetivo: Identificar as barreiras ao comparecimento e adesão de pacientes com DPOC à reabilitação pulmonar. Métodos: Realizou-se uma revisão integrativa da literatura. A localização e seleção dos estudos foi realizada por meio de pesquisa nas bases de dados eletrônicas Medline (acessada pelo PubMed) e Scientific Electronic Library Online (SciELO). A estratégia utilizada foi o cruzamento dos descritores "COPD", "Treatment Adherence and Compliance" e "Rehabilitation". Foram incluídos artigos publicados a partir de 2009 até maio de 2019, escritos nos idiomas inglês, português e espanhol, elegendo como população alvo indivíduos com DPOC que realizaram reabilitação pulmonar e tendo como desfecho a adesão ao tratamento. Resultados: A busca resultou em um achado de 566 artigos nas bases de dados MEDLINE, SCIELO e LILACS, que destes 16 foram incluídos no estudo. Conclusão: As principais barreiras de adesão a reabilitação pulmonar foram tabagismo atual, falta de informação acerca da patologia e da efetividade de tratamento, presença de comorbidades, percepção negativa do estado de saúde, dificuldades de mobilidade e transporte e a falta de apoio social.

Palavras-chave: DPOC; Reabilitação Pulmonar; Barreiras; Adesão.

\begin{abstract}
Background: Pulmonary rehabilitation is extremely important for patients with COPD. However, treatment adherence is low. Understanding the reasons for low compliance is essential for the success of therapy. Aim: To identify barriers to the attendance and adherence of patients with COPD to pulmonary rehabilitation. Methods: An integrative literature review was carried out. The location and selection of the studies was carried out by searching the electronic databases Medline (accessed by PubMed) and Scientific Electronic Library Online (SciELO). The strategy used was the crossing of the descriptors "COPD", "Treatment Adherence and Compliance" and "Rehabilitation". Articles published from 2009 to May 2019, written in English, Portuguese and Spanish, were included, targeting individuals with COPD who underwent pulmonary rehabilitation and having treatment adherence as their outcome. Results: The search resulted in a finding of 566 articles in the MEDLINE, SCIELO and LILACS databases, of which 16 were included in the study. Conclusion: The main barriers to adherence to pulmonary rehabilitation were current smoking, lack of information about the pathology and treatment effectiveness, presence of comorbidities, negative perception of health status, mobility and transport difficulties and lack of social support.
\end{abstract}

Keywords: COPD; Pulmonary Rehabilitation; Barriers; Adherence.

Como citar: Fonte CL, Gomes BB, Cruz SJV Moraes WRA, Neves LMT. Barreiras à adesão ao programa de reabilitação pulmonar de pacientes com doença pulmonar obstrutiva crônica. ASSOBRAFIR Ciênc. 2020;11:e42396. https://doi.org/10.47066/2177-9333. AC. 2020.0016

Submissão em: Dezembro 28, 2020 Aceito em: Maio 05, 2021

Estudo realizado em: Universidade Federa do Pará (UFPA), Belém, PA, Brasil. Aprovação ética: Não se aplica

*Autor correspondente: Carolina Lima da Fonte. E-mail: carollima.fonte@gmail.com 


\section{INTRODUÇÃO}

A Doença Pulmonar Obstrutiva Crônica (DPOC) é uma doença comum, evitável e tratável, caracterizada por sintomas respiratórios persistentes e limitação do fluxo aéreo causadas por exposição significativa a partículas ou gases nocivos. É atualmente a quarta principal causa de morte no mundo, mas é projetada para ser a terceira principal causa de morte até $2020^{1}$. É uma patologia progressiva para qual não há cura, apenas abordagens terapêuticas para aliviar os sintomas e retardar a progressão da doença. Os sintomas mais comuns dessa patologia são: dispneia, produção excessiva de expectoração, tolerância reduzida ao exercício e tosse crônica ${ }^{2,3}$.

A Reabilitação Pulmonar (RP) é uma intervenção não farmacológica, consistindo em um programa multidisciplinar que inclui treinamento físico, educação em saúde e intervenções comportamentais. Esse tratamento é de suma importância para DPOC, pois promove a melhora da dispneia e maior tolerância a exercícios; diminuição da ocorrência de exacerbações e hospitalizações; aumento da participação em atividades físicas e sociais, além da melhora na qualidade de vida relacionada à saúde ${ }^{4-6}$.

Como em outras doenças crônicas, o gerenciamento bem-sucedido dos sintomas depende de muitos fatores, um dos quais é a adesão do paciente ao regime de tratamento. Contudo mesmo a RP sendo um dos tratamentos mais eficazes para o manejo clínico da DPOC, as taxas de adesão e conclusão do tratamento são baixas ${ }^{7,8}$. Compreender os motivos da baixa adesão é imprescindível para o sucesso da terapia. De fato, as consequências da falta de adesão às terapias eficazes são substanciais: piora do prognostico clínico, aumento do número de exacerbações e aumento dos custos com a assistência médica9 ${ }^{9}$. Portanto, são necessárias mais informações sobre os motivos e preditores de não adesão e não participação. Sendo assim, o objetivo dessa revisão é identificar as barreiras que levam ao não comparecimento e não adesão de pacientes com DPOC à reabilitação pulmonar.

\section{MÉTODOS}

Trata-se de um estudo do tipo, revisão integrativa da literatura. Sobre a luz de Whittemore e Knafl:10, este método é um "A revisão integrativa é a mais ampla abordagem metodológica referente às revisões, permitindo a inclusão de estudos experimentais e nãoexperimentais para uma compreensão completa do fenômeno analisado." Nesse contexto os pesquisadores seguiram as recomendações de Souza et al. ${ }^{11}$ com o intuito de garantir a qualidade do estudo, ajudando os autores a melhorar o relato de revisão integrativa.

A localização e seleção dos estudos foi realizada por meio de pesquisa nas bases de dados eletrônicas Medline (acessada pelo PubMed), Scientific Electronic Library
Online (SciELO) e Literatura Latino-Americana e do Caribe em Ciências da Saúde (LILACS). A estratégia utilizada foi o cruzamento dos descritores "COPD", "Treatment Adherence and Compliance" e "Rehabilitation" utilizando o operador booleano "and". Além disso, foi realizada uma pesquisa manual das listas de referência dos estudos incluídos. Foram incluídos artigos publicados que possuíam como população alvo indivíduos com DPOC que realizaram reabilitação pulmonar e tendo como desfecho a adesão ao tratamento. Foram excluídos estudos que não abordaram a temática da adesão ao tratamento, DPOC ou reabilitação pulmonar; artigos do tipo relato de caso e revisões da literatura; e estudos duplicados.

A pesquisa bibliográfica foi realizada de forma independente por 2 pesquisadores. Os mesmos autores selecionaram independentemente estudos potencialmente elegíveis para inclusão. Desacordos entre revisores foram resolvidos por consenso; se não fosse possível chegar a um acordo, solicitava-se a opinião de um terceiro autor e sua decisão era considerada final.

Para análise do material obtido, foi realizada uma leitura dos artigos na íntegra e elaborado um resumo crítico sintetizando os objetivos, a metodologia e as conclusões. Esse processo teve como objetivo a melhor visualização do desenho metodológico do estudo e das possíveis semelhanças e divergências entre esses. Posteriormente, foi feita a exploração do material pela análise detalhada por dois pesquisadores independentes e isoladamente. Por fim, as impressões foram registradas e discutidas entre os autores.

\section{RESULTADOS}

A busca inicial de bancos de dados eletrônicos identificou 560 estudos enquanto a busca manual identificou 6 estudos, totalizando 566 estudos potencialmente relevantes. Destes, foram excluídos 206 por não se encaixarem nos critérios de tipo de estudo. Após a inspeção detalhada dos 360 artigos completos restantes, 16 preencheram os critérios de inclusão da presente revisão. A Figura 1 apresenta um fluxograma do processo de seleção do estudo.

Em relação ao resultado da busca, na base de dados Medline, acessada via Pubmed, foram encontrados 359 resultados, em que destes apenas 16 se enquadraram nos critérios de inclusão deste estudo, na plataforma SCIELO apenas 1 artigo foi encontrado, e o mesmo preencheu os critérios de inclusão. Já na base de dados LILACS não foram encontrados estudos.

Os estudos possuíram um intervalo de tempo de 2006 a 2019. Já em relação ao tipo de estudo foram verificados estudos observacionais de caráter qualitativo e quantitativo, ensaios clínicos e coortes que tiveram como desfecho a análise da adesão de pacientes com DPOC a RP.

As características dos artigos incluídos estão detalhadas na Tabela 1. 


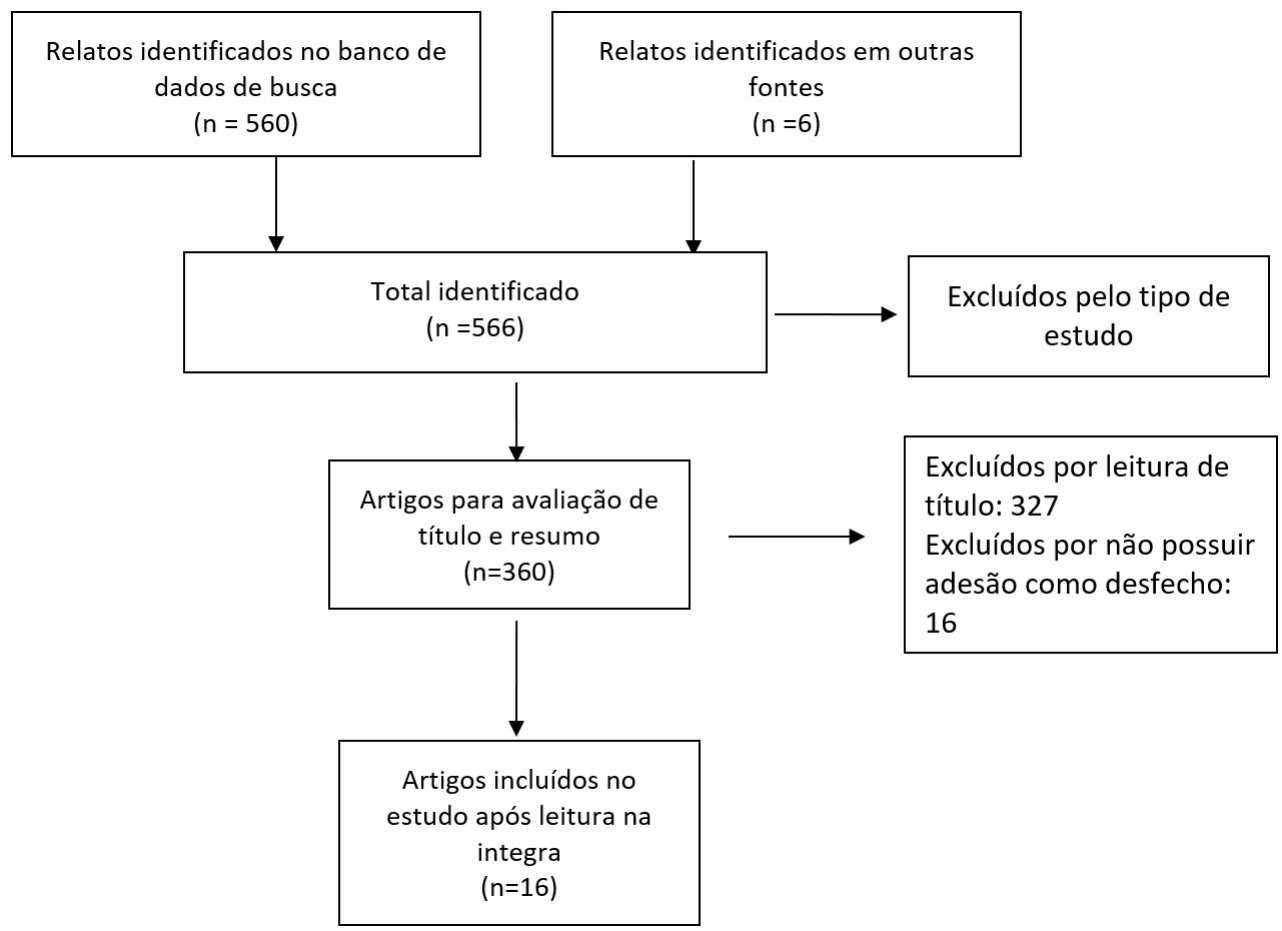

Figura 1. Fluxograma da seleção de artigos.

Tabela 1. Descrição dos estudos incluídos.

\begin{tabular}{|c|c|c|c|}
\hline Estudo & População & Intervenção e Métodos & Desfecho \\
\hline Santana et al. ${ }^{12}$ & $\begin{array}{l}18 \text { pacientes ex-tabagistas } \\
\text { e } 23 \text { tabagistas atuais } \\
\text { (GOLD estádios II-IV). }\end{array}$ & $\begin{array}{c}\text { Estudo prospectivo. Os pacientes } \\
\text { foram submetidos a um programa } \\
\text { de RP com duração de } 12 \text { semanas. } \\
\text { A interrupção da RP antes do } \\
\text { término previsto foi considerada } \\
\text { indicativa de não aderência ao } \\
\text { programa. }\end{array}$ & $\begin{array}{l}\text { A proporção de pacientes não-aderentes } \\
\text { à RP foi maior nos tabagistas do que nos } \\
\text { ex-tabagistas. Observou-se que embora } \\
\text { o tabagismo atual tenha se relacionado } \\
\text { negativamente com a aderência à RP, os } \\
\text { ganhos clínicos e fisiológicos foram similares } \\
\text { nos pacientes ex-tabagistas e tabagistas. }\end{array}$ \\
\hline Kosteli et al. ${ }^{13}$ & $\begin{array}{l}26 \text { indivíduos com DPOC } \\
\text { grave. }\end{array}$ & $\begin{array}{c}\text { Foi aplicado um roteiro de } \\
\text { entrevista semiestruturado com } \\
\text { cada questão destinada a direcionar } \\
\text { os principais componentes que } \\
\text { podem funcionar como barreiras à } \\
\text { adesão. }\end{array}$ & $\begin{array}{l}\text { As principais barreiras observadas são } \\
\text { relacionadas à saúde (fadiga, problemas } \\
\text { de mobilidade, problemas respiratórios), } \\
\text { psicológicos (constrangimento, medo, } \\
\text { frustração / decepção), atitudinal (sentir-se } \\
\text { no controle de sua condição, percepção da } \\
\text { velhice), e motivacional. }\end{array}$ \\
\hline Brown et al. ${ }^{14}$ & $\begin{array}{c}\text { Indivíduos com DPOC } \\
\text { que participaram de } \\
\text { um programa de RP na } \\
\text { Universidade do Alabama } \\
\text { em Birmingham, de } 1996 \\
\text { a } 2013 .\end{array}$ & $\begin{array}{l}\text { Analisou-se um banco de dados } \\
\text { mantido prospectivamente, com } \\
\text { informações sociodemográficas e } \\
\text { comorbidades. Análises univariadas } \\
\text { e multivariadas foram realizadas } \\
\text { para identificar preditores de } \\
\text { conclusão bem-sucedida de PR. }\end{array}$ & $\begin{array}{l}\text { O tabagismo foi o único preditor } \\
\text { independente de não conclusão de PR. }\end{array}$ \\
\hline Hayton et al. ${ }^{15}$ & 711 pacientes com DPOC. & $\begin{array}{l}\text { Foi realizada uma análise } \\
\text { retrospectiva de pacientes com } \\
\text { DPOC, que foram convidados } \\
\text { para participar do PR. Os dados } \\
\text { foram comparados para permitir } \\
\text { a identificação de preditores de } \\
\text { atendimento e aderência. }\end{array}$ & $\begin{array}{l}\text { 31,8\% dos pacientes encaminhados para } \\
\text { RP não compareceram e 29,1\% eram } \\
\text { não aderentes. Os preditores de não } \\
\text { comparecimento foram sexo feminino, } \\
\text { fumante atual e morar sozinho. Os preditores } \\
\text { de não adesão foram extremos de idade, } \\
\text { tabagismo atual, uso de ODP, VEF1, CRQ } \\
\text { pontuação e distância de viagem. Regressão } \\
\text { logística múltipla revelou que LTOT e viver } \\
\text { sozinho foram preditores independentes } \\
\text { de baixa frequência e tabagismo atual, } \\
\text { distância e hospitalizações foram preditores } \\
\text { independentes de baixa adesão. }\end{array}$ \\
\hline
\end{tabular}


Tabela 1. Continuação...

\begin{tabular}{ccc}
\hline Estudo & População & Intervenção e Métodos \\
\hline Boim et al. ${ }^{24}$ & Pacientes com DPOC. & $\begin{array}{c}\text { Um estudo retrospectivo. Avaliou-se } \\
\text { variáveis relacionados à adesão } \\
\text { um PR em um centro da Argentin }\end{array}$ \\
Arnold et al. ${ }^{25}$ & $\begin{array}{c}\text { Vinte pacientes com } \\
\text { DPOC }\end{array}$ & $\begin{array}{r}\text { Estudo qualitativo utilizando } \\
\text { entrevistas semiestruturadas. }\end{array}$
\end{tabular}

Hogg et al. ${ }^{26} \quad 1114$ pacientes com DPOC

Sabit et al. ${ }^{15} \quad 239$ pacientes com DPOC

Keating et al. ${ }^{14} \quad 19$ adultos com DPOC que se recusaram a participar e 18 adultos com

DPOC que não haviam completado um programa de reabilitação pulmonar.

Oates et al. ${ }^{27}$ 24 pacientes com DPOC

Braeken et al. ${ }^{20}$ 518 pacientes com DPOC

Sahin e Naz ${ }^{31}$
Coorte onde foram avaliados a efetividade, participação e conclusão de um programa de reabilitação pulmonar integrado, composto por atendimento hospitalar e ambulatorial. Preditores de atendimento, conclusão e eficácia foram procurados.

Estudo Retrospectivo. Foram investigados quais fatores, identificáveis a partir de dados hospitalares de rotina, predizem baixa frequência uma vez inscritos em um programa de reabilitação pulmonar.

Desenho qualitativo utilizando entrevistas semiestruturadas.

Desenho qualitativo utilizando entrevistas semiestruturadas.

Estudo transversal. Verificou por meio de questionários exclusivos para DPOC o efeito das exacerbações na adesão a RP.

Os pacientes com DPOC encaminhados ao programa de RP foram divididos em dois grupos: os que concluíram o programa foram classificados como grupo 1 e os que não concluíram foram classificados como grupo 2, e seus dados foram comparados.
Desfecho

O baixo nível de adesão estava relacionado com a distância da casa até o centro de saúde e a disponibilidade de renda econômica ou trabalho social.

A influência do médico encaminhador foi o fator chave para o início do programa de reabilitação pulmonar. A adesão contínua a RP foi influenciada por um senso de apoio do grupo e aumento da autoconfiança. A falta de apoio social em casa e a superação do esforço de conviver com a DPOC para atendimento foram citadas como influências negativas na continuidade da adesão.

Os pacientes encaminhados por médicos de clínica geral foram associados com menor taxa de conclusão da reabilitação Pulmonar. A presença de depressão e Dispneia grau 4 e 5 no MRC também foram associados com baixa taxa de adesão.

A participação nos programas de reabilitação Pulmonar é influenciada de forma independente pelo tabagismo, grau de falta de ar, frequência de internações hospitalares, duração do programa e tempo de viagem.

A falta de informação quanto aos benefícios da Reabilitação Pulmonar e do exercício físico foram citados por pacientes que optaram por não participar.

Problemas de mobilidade urbana, distância do centro e saúde e custo de transporte foram citados tanto por aqueles que optaram por não participar e aqueles que não completaram.

Idade avançada, presença de comorbidades, fadiga, e falta de apoio social também foram relatados.

Limitações de saúde, suporte social, dificuldades de transporte e financeiras e as características do programa impactam a capacidade dos pacientes de comparecer à RP. As intervenções que abordam essas barreiras interpessoais, intrapessoais e estruturais são necessárias para facilitar a adesão à RP.

Exacerbações agudas e leves não afetam no abandono da RP, porém exacerbações graves afetam.

Durante as ligações foram verificadas cinco razões para não aderência a RP: falta de motivação, problemas de transporte, exacerbações da DPOC, problemas relacionados ao trabalho e hospitalização (por razões diferentes de exacerbação da DPOC). 
Tabela 1. Continuação...

\begin{tabular}{|c|c|c|c|}
\hline Estudo & População & Intervenção e Métodos & Desfecho \\
\hline McCarron et al. ${ }^{19}$ & 281 pacientes com DPOC & $\begin{array}{l}\text { Um questionário estruturado } \\
\text { composto de barreiras e } \\
\text { facilitadores foi aplicado aos } \\
\text { pacientes que não compareceram } \\
\text { e aqueles que compareceram, mas } \\
\text { não concluíram o programa de RP. }\end{array}$ & $\begin{array}{c}\text { Idade, sexo e tabagismo são fatores que } \\
\text { afetam as taxas de frequência e conclusão } \\
\text { dos pacientes atendidos em RP. A taxa de não } \\
\text { comparecimento foi de } 42 \% \text {. }\end{array}$ \\
\hline Moore et al. ${ }^{30}$ & 24 pacientes com DPOC & $\begin{array}{c}\text { Um estudo qualitativo. Entrevistas } \\
\text { semiestruturadas em pacientes que } \\
\text { não completaram ou recusaram o } \\
\text { tratamento. }\end{array}$ & $\begin{array}{l}\text { Verificou-se que existiam dois grandes } \\
\text { grupos de barreiras: as de aceitabilidade } \\
\text { (expectativas e informações sobre RP; } \\
\text { dificuldades de acesso devido a limites } \\
\text { geográficos ou tempo; dificuldades em } \\
\text { priorizar o tratamento; crenças contrárias } \\
\text { sobre o papel e a segurança do exercício; } \\
\text { medos sobre críticas, exposição e } \\
\text { inadequação. E as barreiras de viabilidade } \\
\text { (comorbidades e dependência de cuidador). }\end{array}$ \\
\hline Oates et al. ${ }^{7}$ & 455 pacientes com DPOC & $\begin{array}{c}\text { Uma análise transversal de um } \\
\text { banco de dados de pacientes com } \\
\text { DPOC em um programa de RP } \\
\text { ambulatorial. }\end{array}$ & $\begin{array}{c}\text { Verificou-se que a baixa adesão está } \\
\text { associada à limitação da capacidade funcional } \\
\text { e ao tabagismo atual, enquanto a moderada } \\
\text { adesão está associada à desvantagem } \\
\text { socioeconômica. }\end{array}$ \\
\hline Almadana et al. ${ }^{22}$ & 83 pacientes com DPOC & $\begin{array}{l}\text { Estudo prospectivo foi realizado } \\
\text { entre fevereiro e novembro } \\
\text { de } 2015 \text { em } 83 \text { pacientes com } \\
\text { DPOC inscritos em um programa } \\
\text { ambulatorial de } 36 \text { sessões de } \\
\text { treinamento de força + resistência. }\end{array}$ & $\begin{array}{c}\text { As principais causas de não adesão estão } \\
\text { relacionadas à motivação e ao transporte. } \\
\text { Os pacientes que abandonam possuíam } \\
\text { os piores testes funcionais, além de mais } \\
\text { exacerbações, uso de corticosteróides e } \\
\text { tabagismo. }\end{array}$ \\
\hline
\end{tabular}

\section{DISCUSSÃO}

Este estudo buscou verificar as barreiras de adesão a RP de pacientes com DPOC, desta forma, foi possível observar que os fatores que envolvem a não permanência dos pacientes ao tratamento perpassam fatores sociais, econômicos, psicológicos e também relacionadas a doença.

A DPOC pode ocasionar vários sintomas que contribuem para diminuição da funcionalidade e impactam diretamente na qualidade de vida do paciente. Em vista disso, a Reabilitação Pulmonar atua na melhora dessa sintomatologia, porém, para que o tratamento seja mais efetivo são fundamentais o comparecimento ao atendimento e a efetiva adesão. Entretanto, a realidade encontrada pelos serviços que oferecem a reabilitação é de baixa adesão dos participantes ao tratamento, comprometendo a eficácia da terapêutica, uma vez que a participação em menos de $50 \%$ das sessões não levam a benefícios perceptíveis ao paciente ${ }^{4,12,13}$.

A definição de não adesão ao tratamento é variada, alguns estudos a definem como não comparecimento a nenhuma sessão de fisioterapia ${ }^{14}$, também como a frequência menor que $50 \%$ as sessões recomendadas ${ }^{15,16}$, ou até mesmo como o abandono do tratamento em qualquer fase antes de sua conclusão ${ }^{18}$. Com isso, tornase difícil a padronização de estudos onde a adesão tenha sido definida seguindo os mesmos conceitos, porém como o objetivo do presente estudo é identificar as barreiras da adesão foi entendido que a não padronização da mesma não seria um viés para o alcance do objetivo final.
Foram identificadas diversas barreiras à aderência ao programa de reabilitação pulmonar, sejam relatadas pelo usuário ou pelo profissional, dentre elas podemos citar o tabagismo, barreiras próprias da doença (exacerbações e hospitalizações frequentes); barreiras relacionadas à falta de informação quanto à patologia e quanto aos efeitos da RP, restrições de tempo, problemas de transporte, motivação para a realização, grande distância de deslocamento até o local do atendimento, além de falta de apoio familiar.

Possuir uma história de tabagismo atual predispõe diretamente ao risco de desistência, pois pacientes que não obtém sucesso na cessação do tabagismo, consequentemente, possuirão baixo sucesso em um programa de reabilitação devido à má gestão comportamental. Brown et al. ${ }^{18}$ realizaram uma coorte prospectiva e observaram que o tabagismo foi o único preditor independente de abandono da Reabilitação Pulmonar. Sendo assim, a cessação do tabagismo pode promover maior aderência ao tratamento.

De maneira semelhante, Santana et al. ${ }^{17}$ realizaram um estudo com 18 pacientes ex-tabagistas e 23 tabagistas atuais avaliando sua adesão a um programa de reabilitação pulmonar de 12 semanas, em que foi observado a maior aderência ao programa de RP no grupo de pacientes extabagistas quando comparado ao grupo de tabagistas atuais. Além disso, os autores também evidenciaram que os ganhos clínicos e fisiológicos proporcionados pela reabilitação são similares em ambos os grupos, sugerindo que intervenções devem focar na educação em saúde 
para a cessação do tabagismo, visto os possíveis ganhos advindos do tratamento.

Em concordância a esses achados, o estudo de McCarron et al. ${ }^{19}$ que incluiu 281 pacientes e tinha como objetivo identificar barreiras e facilitadores ao comparecimento e conclusão à um programa de $\mathrm{RP}$, demonstrou que o status de fumante contribuía diretamente para o não comparecimento e para a não conclusão ao programa. Os autores acreditam que esse achado está associado ao estado de saúde, pois os fumantes são mais propensos a sofrer dispneia e outros efeitos colaterais físicos, limitando sua participação nos exercícios. Além disso, há uma percepção de culpabilidade e vergonha desses pacientes, atuando como barreira

A presença de sintomatologia intensa e exacerbações frequentes podem influenciar a adesão ao tratamento. Sabit et al. ${ }^{15}$ observaram uma relação entre a baixa frequência de comparecimento à reabilitação com o alto escore de dispneia do MRC entre 4 e 5 . Em vista disso, pacientes que apresentam dispneia - tanto em repouso quanto com esforço - apresentavam mais dificuldades de lidar com a demanda fisiológica da RP, e por conta disso, abandonavam o tratamento.

O estudo de Braeken et al. ${ }^{20}$ que objetivava avaliar o impacto de exacerbações leves, moderadas e graves no abandono e nos resultados da RP, identificou por meio da avaliação de 466 pacientes que a ocorrência de exacerbações leves e moderadas não afetam a taxa de conclusão do programa, entretanto as exacerbações graves estão associadas a aumento do abandono. Foi observado também que os pacientes que concluem a reabilitação, mesmo após a ocorrência de uma exacerbação grave, respondem positivamente ao tratamento.

O estado de saúde percebido pelo paciente também é um preditor importante. Selzler et al. ${ }^{21}$ observaram que o status de saúde no início da RP foi um importante preditor de abandono, com aqueles que abandonaram o tratamento tendo percepções piores do estado de saúde. Os autores apontam que, por vezes, o comprometimento percebido pode ter maior probabilidade de influenciar a conclusão da reabilitação do que o comprometimento pulmonar real.

Em consonância a isso Almadana et al. ${ }^{22}$ constatou em seus estudos que pacientes não aderentes a RP possuíam um perfil mais grave da doença, sendo caracterizada por mais exacerbações, maior capacidade vital forçada (que pode ser associada a hiperinsuflação pulmonar, característico de paciente com DPOC) e piores resultados em testes de capacidade funcional.

Além dos problemas de saúde, Kosteli et al. ${ }^{23}$ relatou que impacto psicológico das limitações físicas associadas à DPOC, incluindo vergonha, medo e frustração / decepção também reduzem a adesão. Os profissionais de saúde devem levar em consideração o estado psicológico dos indivíduos e explorar maneiras de ajudá-los a perceber o impacto positivo da RB em sua doença.
Problemas de mobilidade, transporte público e baixo nível econômico também são apontados como fatores limitantes para a adesão à programas de RP. Por meio da análise de dados hospitalares, notou-se que o acesso aos programas de RP é prejudicado para pacientes que possuem mobilidade reduzida e com poucos recursos financeiros devido às dificuldades de transportes e de deslocamento até o local de atendimento, sendo preditores de faltas ao atendimento ${ }^{15,21}$. Boim et al. ${ }^{24}$ pontuaram que um maior poder socioeconômico permite maior acesso à medicação e apoio necessário para a realização da RP.

A falta de informação acerca dos benefícios da RP influencia o comparecimento dos pacientes ao serviço. No estudo realizado por Arnold et al. ${ }^{25}$, o incentivo médico foi o principal fator para determinar o ingresso de um paciente na RP, pois os pacientes tendem a confiar nas informações repassadas por esse profissional. Nesse estudo, os pacientes responderam de maneira positiva ao início da intervenção quando seus médicos proporcionavam informações sobre os benefícios da RP.

Por meio de entrevistas semiestruturadas, Keating et al. ${ }^{14}$ analisaram os motivos que levaram 19 pacientes com DPOC à não conclusão de um programa de RP. Foi demonstrado que 11 dos 19 participantes optaram por não participar do programa pois não achavam que a RP traria benefícios a sua saúde em geral. Portanto, pacientes que não conhecem os benefícios promovidos pela RP são mais propensos a não aderir a esse tratamento.

Sendo assim, a adoção de estratégias de educação em saúde é primordial para o aumento da adesão à RP e, consequentemente, ao sucesso do tratamento. A educação em saúde em DPOC é de suma importância para que o paciente esteja preparado para lidar com o manejo da doença e possua maior compreensão acerca dos tratamentos necessários.

Desse modo, ela promove a participação ativa do indivíduo em sua saúde; ajuda o paciente e familiares a lidarem com a doença e suas consequências; facilita a compreensão de alterações físicas e psicológicas decorrentes da condição patológica ${ }^{25}$. Assim, os pacientes têm o poder de participar ativamente de seus próprios cuidados de saúde, o que pode promover a adesão à terapia.

A falta de apoio social ao tratamento foi vista também como uma barreira a essa adesão, pacientes que não contavam com esse apoio e motivação foram mais propensos a não aderir ao programa de RP ${ }^{16}$. Além disso, é importante que o paciente gostar do programa, ou seja, dos sentimentos de prazer e realização durante o mesmo, pois, como os pacientes com DPOC possuem incidência de depressão elevada, atividades que promovam bem-estar são de suma importância ${ }^{25,26}$.

Oates et al. ${ }^{27}$ descreveu em seus estudos alguns facilitadores para adesão ao tratamento, os mesmos foram relatados pelos pacientes durante as entrevistas estruturadas desenvolvidas. O facilitador de maior 
relevância foi a percepção de melhora do estado de saúde, de forma que os participantes se sentiram motivados a concluir suas sessões ao perceber que podiam respirar melhor, andar mais ou perder peso. Além disso, outros facilitadores foram citados como o apoio familiar e incentivo emocional, ressaltando também a importância da socialização com outros participantes do programa.

Além disso, outro fator de não adesão encontrado foi o que a desvantagem socioeconômica, no estudo de Oates et al. ${ }^{7}$ foi verificado por meio de quinze variáveis, como renda familiar, escolaridade e valor médio de habitação, que populações menos favorecidas tinham menos chance de concluir o plano de RP a longo prazo, fato que verificado em um estudo ${ }^{28}$ semelhante realizado em centro de Reabilitação Cardíaca na qual demonstrou que pacientes que possuíam associação de dificuldades socioeconômicas e características da doença possuíam menos adesão ao tratamento, logo maior atenção para a manutenção da frequência

Outra variável verificada foram os fatores psicossociais adquiridas pelos pacientes, como ansiedade e depressão. Pierbon et al. ${ }^{4}$ em seu estudo relata que esses pontos influenciam diretamente na execução da terapia comportamental, medicamentosa e na execução dos exercícios, e por consequência, uma piora no estado funcional do paciente. De acordo com um estudo ${ }^{29}$, que foi realizado com 75 pacientes com DPOC estável, notouse que 32 destes apresentavam depressão e ansiedade concomitantemente, e apenas 9 pacientes apresentavam tais repercussões de forma isolada, sendo esses com graus de DPOC elevado.

De forma semelhante, a motivação pessoal associada ao comparecimento à RP foi um ponto destacado por estudos ${ }^{30,31}$ que demostra a falta de interesse ao tratamento sem um motivo específico. Mathar et al. ${ }^{32}$ descreve que a falta de motivação está relacionada as percepções do paciente sobre a RP e sobre a saúde, da falta de relevância da atividade física e de ter outras prioridades na vida.

Em vista disso, a partir da identificação das principais barreiras à adesão ao programa de reabilitação pulmonar é possível definir as melhores estratégias para minimizar este quadro. Com isso, observa-se a importância da aplicação de estratégias de educação em saúde para uma maior conscientização acerca da patologia e suas possibilidades de tratamento; da adoção de medidas que visem a cessação do tabagismo, assim como o encaminhamento desses pacientes aos centros de referência para a realização da Terapia Cognitivo comportamental; da disponibilização de transportes para facilitar o deslocamento dos pacientes até o local do tratamento; da promoção de atividades que incluam os membros da família para promover um suporte social, assim como a promoção de atividades que estimulem o prazer e bem-estar dos pacientes; e, por fim, da adoção de estratégias que utilizem o meio digital, como a telerreabilitação, a fim de aumentar a proximidade do paciente com o terapeuta e o centro de tratamento, fornecendo informações importantes e proporcionando monitoramento adequado da patologia.

\section{CONCLUSÃO}

As principais barreiras de adesão a reabilitação pulmonar foram tabagismo atual, falta de informação acerca da patologia e da efetividade de tratamento, presença de comorbidades, percepção negativa do estado de saúde, dificuldades de mobilidade e transporte e a falta de apoio social. A partir da identificação dessas barreiras é possível desenvolver estratégias para promover o aumento da adesão ao programa de reabilitação pulmonar, o que pode gerar maior efetividade desta terapêutica, refletindo em redução da mortalidade e aumento da qualidade de vida dos pacientes com DPOC.

\section{FONTE DE FINANCIAMENTO}

Nada a declarar.

\section{CONFLITO DE INTERESSES}

Nada a declarar.

\section{REFERÊNCIAS}

1. Alvar A, Decramer M, Frith P. Global Initiative for Chronic Obstructive Lung Disease. Pocket guide to COPD diagnosis, management and prevention. Glob Initiat chronic Obstr lung Dis [Internet]. 2019 [cited 2019 Dec 15];1-42. Available from: www.goldcopd.org.

2. Keating A, Lee A, Holland AE. What prevents people with chronic obstructive pulmonary disease from attending pulmonary rehabilitation? A systematic review. Chron Respir Dis. 2011 Maio;8(2):89-99. http://dx.doi. org/10.1177/1479972310393756. PMid:21596892.

3. Miravitlles M, Ribera A. Understanding the impact of symptoms on the burden of COPD. Respir Res. 2017;18(1):67. http://dx.doi.org/10.1186/s12931-017-05483. PMid:28431503.

4. Pierobon A, Sini Bottelli E, Ranzini L, Bruschi C, Maestri R, Bertolotti $G$, et al. COPD patients' self-reported adherence, psychosocial factors and mild cognitive impairment in pulmonary rehabilitation. Int J Chron Obstruct Pulmon Dis. 2017;12:2059-67. http://dx.doi.org/10.2147/COPD.S133586. PMid:28790808.

5. Zuwallack RL. How do we increase activity and participation in our patients?. Semin Respir Crit Care Med. 2009;30(6):70812. http://dx.doi.org/10.1055/s-0029-1242640.

6. WalshJR, PeggJ, Yerkovich ST, Morris N, McKeough ZJ, Comans $\mathrm{T}$, et al. Longevity of pulmonary rehabilitation benefit for chronic obstructive pulmonary disease-health care utilisation in the subsequent 2 years. BMJ Open Respir Res. 2019;6(1):e000500. http://dx.doi.org/10.1136/bmjresp-2019-000500. http:// dx.doi.org/10.1136/bmjresp-2019-000500.

7. Oates GR, Hamby BW, Stepanikova I, Knight SJ, Bhatt SP, Hitchcock J, et al. Social Determinants of Adherence to Pulmonary Rehabilitation for Chronic Obstructive 
Pulmonary Disease. COPD. 2017;14(6):610-7. http://dx.doi. org/10.1080/15412555.2017.1379070. PMid:29020525.

8. Boutou AK, Tanner RJ, Lord VM, Hogg L, Nolan J, Jefford $\mathrm{H}$, et al. An evaluation of factors associated with completion and benefit from pulmonary rehabilitation in COPD. BMJ Open Respir Res. 2016;1:e000051. doi: http://dx.doi.org/10.1136/ bmjresp-2014-000051.

9. Hogea SP, Tudorache E, Fildan AP, Fira-Mladinescu O, Marc $\mathrm{M}$, Oancea C. Risk factors of chronic obstructive pulmonary disease exacerbations. Clin Respir J. 2020;14(3):183-97. http:// dx.doi.org/10.1186/s12931-019-1262-0. PMid:31814260.

10. Whittemore $\mathrm{R}, \mathrm{Knafl} \mathrm{K}$. The integrative review: updated methodology. J Adv Nurs. 2005;52(5):546-53. http://dx.doi. org/10.1111/j.1365-2648.2005.03621.x. PMid:16268861.

11. Souza MT, Silva MD, Carvalho R. Integrative review: what is it? How to do it? Einstein (Sao Paulo). 2010 Mar;8(1):1026. http://dx.doi.org/10.1590/s1679-45082010rw1134. PMid:26761761.

12. Nici L. Adherence to a pulmonary rehabilitation program: start by understanding the patient. COPD. 2012;9(5):4456. http://dx.doi.org/10.3109/15412555.2012.718931. PMid:23030584.

13. Bender BG. Nonadherence in chronic obstructive pulmonary disease patients: what do we know and what should we do next?. Curr Opin Pulm Med. 2014;20(2):132-7. http://dx.doi. org/10.1097/MCP.0000000000000027.

14. Keating A, Lee AL, Holland AE. Lack of perceived benefit and inadequate transport influence uptake and completion of pulmonary rehabilitation in people with chronic obstructive pulmonary disease: a qualitative study. J Physiother. 2011;57(3):183-90. http://dx.doi.org/10.1016/S18369553(11)70040-6. PMid:21843834.

15. Sabit R, Griffiths TL, Watkins AJ, Evans W, Bolton CE, Shale DJ, et al. Predictors of poor attendance at an outpatient pulmonary rehabilitation programme. Respir Med. 2008;102(6):819-24. http://dx.doi.org/10.1016/j. rmed.2008.01.019. PMid:18337077.

16. Hayton C, Clark A, Olive S, Browne P, Galey P, Knights $\mathrm{E}$, et al. Barriers to pulmonary rehabilitation: characteristics that predict patient attendance and adherence. Respir Med. 2013;107(3):401-7. http://dx.doi.org/10.1016/j. rmed.2012.11.016. PMid:23261311.

17. Santana VTS, Squassoni SD, Neder JA, Fiss E. Influência do tabagismo atual na aderência e nas respostas à reabilitação pulmonar em pacientes com DPOC. Rev Bras Fisioter. 2010;14(1):16-23. http://dx.doi.org/10.1590/S141335552010000100004. PMid:20414557.

18. Brown AT, Hitchcock J, Schumann C, Wells JM, Dransfield MT, Bhatt SP. Determinants of successful completion of pulmonary rehabilitation in COPD. Int J Chron Obstruct Pulmon Dis. 2016;11:391-7. http://dx.doi.org/10.2147/COPD. S100254. PMid:26955269.

19. McCarron EP, Bailey M, Leonard B, McManus TE. Improving the uptake: barriers and facilitators to pulmonary rehabilitation. Clin Respir J. 2019;13(10):624-9. http://dx.doi. org/10.1111/crj.13068. PMid:31344320.

20. Braeken DCW, Spruit MA, Houben-Wilke S, Smid DE, Rohde GGU, Wouters EFM, et al. Impact of exacerbations on adherence and outcomes of pulmonary rehabilitation in patients with COPD. Respirology. 2017;22(5):942-9. http:// dx.doi.org/10.1111/resp.12987. PMid:28139873.
21. Selzler AM, Simmonds L, Rodgers WM, Wong EYL, Stickland MK. Pulmonary rehabilitation in chronic obstructive pulmonary disease: predictors of program completion and success. COPD J Chronic Obstr Pulm Dis. 2012;9(5):538-45. http://dx.doi.org/10.3109/15412555.2012.705365.

22. Almadana Pacheco $V$, Pavón Masa M, Gómez-Bastero Fernández AP, Muñiz Rodríguez AM, Tallón Moreno R, Montemayor Rubio T. Patient Profile of Drop-Outs From a Pulmonary Rehabilitation Program. Arch Bronconeumol. 2017;53(5):257-62. http://dx.doi.org/10.1016/j. arbres.2016.06.010. PMid:27480263.

23. Kosteli MC, Heneghan NR, Roskell C, Williams SE, Adab P, Dickens AP, et al. Barriers and enablers of physical activity engagement for patients with COPD in primary care. Int J COPD. 2017;12:1019-31. http://dx.doi.org/10.2147/COPD. S119806. PMid:28405162.

24. Boim C, Caberlotto O, Storni M, Cortiñaz M, Monti F, Khoury M. Adherencia a un programa interdisciplinario de rehabilitación respiratoria. Medicina (B Aires). 2014;74(2):104-9. PMid:24736252.

25. Arnold E, Bruton A, Ellis-Hill C. Adherence to pulmonary rehabilitation: a qualitative study. Respir Med. 2006;100(10):1716-23. http://dx.doi.org/10.1016/j. rmed.2006.02.007. PMid:16554147.

26. Hogg L, Garrod R, Thornton H, McDonnell L, Bellas H, White P. Effectiveness, attendance, and completion of an integrated, system-wide pulmonary rehabilitation service for COPD: prospective observational study. COPD. 2012;9(5):546-54. http://dx.doi.org/10.3109/15412555.201 2.707258. PMid:23030586.

27. Oates GR, Niranjan SJ, Ott C, Scarinci IC, Schumann C, Parekh $\mathrm{T}$, et al. Adherence to pulmonary rehabilitation in COPD: a qualitative exploration of patient perspectives on barriers and facilitators. J Cardiopulm Rehabil Prev. 2019;39(5):3449. http://dx.doi.org/10.1097/HCR.0000000000000436. PMid:31348127.

28. Gaalema DE, Savage PD, Rengo JL, Cutler AY, Elliott RJ, Priest JS, et al. Patient characteristics predictive of cardiac rehabilitation adherence. J Cardiopulm Rehabil Prev. 2017;37(2):103-10. http://dx.doi.org/10.1097/ HCR.0000000000000225. PMid:28033166.

29. Doyle T, Palmer S, Johnson J, Babyak MA, Smith P, Mabe $S$, et al. Association of anxiety and depression with pulmonaryspecific symptoms in chronic obstructive pulmonary disease. Int J Psychiatry Med 2013;45(2):189-202. http://dx.doi. org/10.2190/PM.45.2.g.

30. Moore L, Hogg L, White P. Acceptability and feasibility of pulmonary rehabilitation for COPD: a community qualitative study. Prim Care Respir J. 2012;21(4):419-24. http://dx.doi. org/10.4104/pcrj.2012.00086. PMid:23135218.

31. Sahin $\mathrm{H}, \mathrm{Naz} \mathrm{I}$. Why are COPD patients unable to complete the outpatient pulmonary rehabilitation program? Chron Respir Dis. 2018;15(4):411-8. http://dx.doi. org/10.1177/1479972318767206. PMid:29673263.

32. Mathar $\mathrm{H}$, Fastholm $P$, Lange $P$, Larsen NS. Why do patients decline participation in offered pulmonary rehabilitation? A qualitative study. Clin Rehabil. 2017;31(12):1674-83. http:// dx.doi.org/10.1177/0269215517708821. PMid:28523990. 